\title{
Effectiveness of Mirror Therapy for Muscle Strength in Ischemic Stroke Patients with Hemiparesis
}

\author{
Dedi Irawandi \\ Medical Surgical Nursing \\ Email: yudisadiaz@gmail.com
}

\author{
Sandi Alfa \\ Medical Surgical Nursing \\ Email:yudisadiaz@gmail.com
}

\begin{abstract}
Background: Stroke is clinical symptoms which disturbances in blood circulation to the brain caused either local or global malfunctioning that occurs suddenly and rapidly progressive that usually caused hemiparesis in stroke patients. Late and inappropriate exercises management may cause permanent disability. The range of motion exercises and early mobilization in stroke patients may reduce the risk of disability. The one of rehabilitation and intervention is muscle strength exercise by stimulating the nerves and improve the functional status of the motor/muscle strength by using mirror therapy. Method: These research based on the literature review (systematic review) of international journals, which is use quasiexperiment, one group pre-post test design. These research using purposive sampling with 24 respondents, while the research instruments using observation sheet those are muscle strength scale and visual imagery scale, the exercise guide sheets and mirror as media. The research analysis using univariate and bivariate analysis. A bivariate analysis using Wilcoxon test. Results: The results of these studies indicate that there is increasing in average muscle strength after mirror therapy exercises five times a day for 7 days evidenced by prior intervention the muscles strength mean of upper extremities is 2:12 (0.45) and the muscle strength mean of the lower extremities is 2:12 (0:45). After the intervention, the muscle strength mean of upper extremities became $3.83(0.56)$ and the muscle strength mean of the lower extremities became 4.00 (0.66). Based on the results of bivariate analysis obtained the calculated value (4369) and significance levels $(p)<0.05$. Conclusion: The results of the journal's review can be concluded that there is significant differences are muscle strength before and after mirror therapy exercise in ischemic stroke patients with hemiparesis.
\end{abstract}

Keywords: muscle strength, mirror therapy, ischemic stroke, hemiparesis.

\section{INTRODUCTION}

Stroke is clinical symptoms by interruption of blood circulation to the brain, causing local or global malfunctioning that occurs suddenly, and rapidly progressive. According to the data stated that every year there were 15 million people worldwide suffered from a stroke, which is 6 million people suffered deaths and 6 million people suffered permanent disability. The death rates will continue increased from 6 million in 2010 to 8 million in 2030 .

[1]Stroke accounts for 1 in 18 deaths in the United States. In 2009 the prevalence of stroke was 6.4 million. Approximately 795,000 people experience a new stroke, 610,000 of them experiencing first attacks and 185,000 recurrent attacks and the cost of the treatment in 2009 is about 68.9 billion US dollar for health and rehabilitation of stroke[1]. Generally, stroke divided into two types: ischemic stroke and hemorrhagic stroke. The incidence of ischemic stroke approximately $85 \%$ of all stroke cases. In Indonesia Government Hospital, stroke is the leading cause of death, the third cause of death and the main cause of disability in the hospital. Based on the Basic Health Research in 2013, the prevalence of stroke in Indonesia was 7 of 1,000 populations, and who have been diagnosed by health workers was 12.1 of 1,000 populations. Also, it had been estimated that stroke is the cause of death in hospital 15\%, with impairment reached $65 \%$.

In stroke patients, $70 \%-80 \%$ experienced hemiparesis (muscle weakness on the one side of the body) by $20 \%$ could improve the motor function while about $50 \%$ had residual symptoms such as motor function disorders/muscle weakness in the extremities. If they do not get a good therapy choice in post-stroke intervention and rehabilitation. When hemiparesis patients did not get optimal management about 30\% - 60\% they will experience extremities full function loss within six months of post-stroke.

Interventions for healing that could be performed in addition to medication or drugs is physiotherapy/ exercises such as; weightlifting, balance and resistance training, hydrotherapy, and Range Of Motion (ROM) exercise. Among those, ROM is often performed in the rehabilitation process of stroke patients either active or passive and can be performed in the hospital.

In addition to rehabilitation therapy ROM, either unilateral or bilateral, the mirror therapy is alternative that can be applied and combined in stroke patients to improve the functional status of the sensory motor. Mirror therapy is non-invasive intervention, directly related to the motor system by train/stimulate the sensory ipsilateral or contralateral sensory motor cortex lesions. This therapy relies on the interaction of 
visual-motor perception to improve the movement of the muscle weakness on one side of the body or hemiparesis.

Mirror therapy exercises is a rehabilitation or exercise that train the imagery or patient's motor imagination. The mirror will provide visual stimulation to the brain (cerebral motor nerves i.e. ipsilateral or contralateral for hemiparesis movement) and the hemiparesis will be observed and imitated like the one in the mirror[2].

Several studies had conducted by scanning the brain and found that during mirror therapy, the active area of this trial is the prefrontal cortex area premotor cortex, parietal cortex, and cerebellum which is the area of motor movements. Therefore repetitive stimulation increased muscle strength and prevent more damage of the neuromuscular and prevent its spread to other areas[2]. Cortex area of human brain which called F5 with respect to its role in motor movements and visuomotor that send signals when observing, imitating or copying the certain action of what is observed so that the person imagination activated the movement area same as the actual movement.Ease of Use[3].

\section{RESEARCH METHODS}

The method of this study using literature review journals systematic review. These study determined whether there were differences in muscle strength before and after mirror therapy in patients with ischemic stroke. The result of one journal research study, there were 24 patients with criteria diagnosed with ischemic stroke who had passed the critical phase and experience hemiparesis or weakness of one side of the body. The patients got muscle strength measurements, aged adults (18-65 years), composmentis consciousness (GCS = E4V5M6), got the first attack, muscle strength range is $1-3$, were not impaired in hearing and sight (VIS scale: 4), fluids and electrolytes within the normal range. After the desired patients criteria were obtained, patients will be given a range of motion exercises to train the muscle strength five times a day for seven days in the part of the healthy body, then the patient was advised to look in the mirror and imagine as if the sick body part moves like the healthy body.

The independent variable in this study is muscle strength exercises whereby mirror and the dependent variable is muscle strength of upper-lower extremity which had hemiparesis, while confounding variables were age, sex and time of treatment in hospital (admission time). From journal review, one of the sampling methods is using non-probability sampling that is purposive sampling-sample selection technique which is based on the specified purpose of the researcher.

\section{RESULT}

The result of journal review had done as follows: based on journals reviewed, the characteristics of ischemic stroke respondents with hemiparesis is $n=24$, the age of the that the most experienced ischemic stroke is in 56-65 years old with $45.8 \%$. Based on gender can be seen more women than men with a percentage of $54.2 \%$. Duration of respondents get first aid in the hospital should be less than 6 hours. From journals reviewed, the average strength of upper muscles after mirror therapy exercise is $2: 12(0: 45)$, while the average of lower muscle strength after mirror therapy exercises was $4.00(0.66)$.

\section{CONCLUSION}

Based on the journals reviewed can be concluded that stroke occured most in 56-65 years old (45.8\%). In older people, the risk of stroke is increased[4]. Stated that every ten years after age 55, the risk of stroke increased is twice. Revealed that in elderly the main artery out of blood vessels is harder, thicker and less elastic as a result of changes in connective tissue in blood vessels which can increased blood pressure. That condition was said as atherosclerosis, which is one risk factor for ischemic stroke. Characteristics of respondents by sex showed that women more suffer from the stroke than men $(54.2 \%)$. The incidence of stroke was different between men and women, which is women more than men after suffering a stroke[5]. However, based on these studies there were no significant differences between men and women regarding: the type of stroke, severity, and case fatality rate. Furthermore, also found there were similarities numbers in mortality in male and female leading by stroke[6].

The risk factors of stroke in older women associated with body fat distribution in which the condition caused by women in menopause[7]. The duration between after attacked and admitted to the hospital and then hospitalized (admission time) also affect the risk of stroke and stroke recovery. Those results showed that all respondents obtained aid treatment at the hospital less than 6 hours. The sooner get help precisely, the risk of cerebral infarction is smaller. Thus, neurological deficit/neurologic damage is less.

The recovery of stroke patients with minimum infarction will faster than the more severe cerebral infarction. The results of stroke treatment will be maximum for cerebral reperfusion if less than 6 hours of admission time. The available time when a person got attacked is 3-6 hours and should get help immediately in the hospital called the golden period. If more than 6 hours the patient will experience the severe disability, because the severity of disability caused by stroke is determined by the appropriate first treatment and the type of stroke.

The duration between first attack and hospitalization (admission time) also affect the risk of stroke and stroke recovery. In the results above, showed that all respondents obtain treatment aid at the hospital less than 6 hours. The sooner get help precisely, the risk of cerebral infarction getting smaller thus neurological deficit/neurologic damage is less.

These systematic review aimed to determine the effectiveness of mirror therapy to muscular strength in ischemic stroke patients with hemiparesis. These interventions can be recommended by room nurses for the management of mobilization and exercises to prevent permanent disability in stroke patients with hemiparesis. These mirror therapy may apply to families who have family strokes and try the exercises at home for the recovery process for the purpose to increase the motor functional status of post-stroke. 


\section{REFERENCES}

[1] S. Facts, "H a rt Hea t Disease isea e a and d ok Stat Statistics ic - 5 Update," 2005.

[2] Y. J. Kang et al., "Upper extremity rehabilitation of stroke : Facilitation of corticospinal excitability using virtual mirror paradigm," pp. 1-8, 2012.

[3] Y. G. Rizzolatti and L. Craighero, "T HE M IRROR N EURON S YSTEM," 2004.

[4] H. Rodgers, J. Greenaway, T. Davies, R. Wood, N. Steen, and R. Thomson, "North East of England," 2004.

[5] R. E. Petrea, A. S. Beiser, S. Seshadri, M. Kellyhayes, C. S. Kase, and P. A. Wolf, "NIH Public Access," vol. 40, no. 4, pp. 1032-1037, 2010.
[6] C. L. Hart, D. J. Hole, and G. D. Smith, "Risk Factors and 20-Year Stroke Mortality in Men and Women in the Renfrew / Paisley Study in Scotland," pp. 19992008, 2007.

[7] A. R. Folsom, R. J. Prineas, S. A. Kaye, and R. G. Munger, "Incidence of Hypertension and Stroke in Relation to Body Fat Distribution and Other Risk Factors in Older Women," vol. 1986, no. January, 1986. 